\title{
Delayed coronary obstruction after lotus transcatheter aortic valve replacement treated with left main stent in stent implantation
}

\author{
Maximilian Krisper ${ }^{1}$, Marco Toselli ${ }^{1,2}$, Tobias Daniel Trippel ${ }^{1}$, \\ Burkert Mathias Pieske ${ }^{1}$, Florian Krackhardt ${ }^{1}$ \\ ${ }^{1}$ Department of Cardiology, Charité - Universitätsmedizin Berlin, \\ Campus Virchow Klinikum, Berlin, Germany \\ ${ }^{2}$ Division of Cardiology, Azienda Ospedaliera Universitaria di Parma, Parma, Italy
}

Transcatheter aortic valve replacement (TAVR) is a validated treatment option for patients presenting with severe aortic valve stenosis and intermediate/high surgical risk. Nevertheless, the procedure may lead to severe complications including coronary obstruction (CO). CO most frequently occurs within minutes after TAVR and, more rarely, up to several days after valve implantation (delayed CO [DCO]) [1]. Reported herein a case of late DCO including a brief discussion of the current literature.

An 84-year old woman presented to the emergency room in cardiogenic shock with severely depressed left ventricular ejection fraction (LVEF 15\%) due to anterior, apical and septal akinesia.

Three months prior to this event, the patient had undergone transfemoral TAVR (Boston Scientific Lotus, $25 \mathrm{~mm}$ ) with the high implantation technique [2]. Before the procedure, coronary artery disease was ruled out and LVEF was $50 \%$. The patient had to be stabilized with extensive medical hemodynamic support. The transesophageal echocardiography showed an optimal TAVR device position without paravalvular leak and normal transvalvular gradient. A coronary angiogram was urgently performed showing subtotal ostial narrowing of the left main coronary artery originating from the $25 \mathrm{~mm}$ Lotus-device with Thrombolysis in Myocardial Infarction (TIMI) III flow in the left anterior descending, and circumflex arteries. The slit-like orifice seemed to be obstructed by calcific debris of the native valve which had pressed into the aortic sinus. A challenging but successful implantation of a drug-coated stent through the device meshes was performed (Coroflex ISAR $3.5 \times 8 \mathrm{~mm}$ ) after multiple angioplasties with non-compliant balloons. However, because of the elevated compression force the angiographic result was sub-optimal. Therefore, in order to minimize the recoil a decision was made to implant a second stent (drug-eluting stent Xience $3.5 \times 8 \mathrm{~mm}$ ). In the current literature this practice is debatable and might increase the risk of deleterious effects, but the final outcome was good (Fig. 1). Catecholamines were rapidly discontinued and LVEF improved from $15 \%$ to $40 \%$. At 6 -month follow up no angina nor dyspnoea were detected, the patient was ambulatory and doing well.

Coronary obstruction is a rare, potentially dramatic complication of TAVR and the incidence of acute $\mathrm{CO}$ (within a few minutes from the procedure) is reported to be $<1 \%[1,3-5]$. The current literature provides limited clinical data regarding DCO. In a recent large series by Jabbour et al. [1] the incidence of DCO, both early and late ( $>7$ days), was $0.22 \%$ but might be under-diagnosed because of undetected cases and sudden cardiac deaths. Women are more often affected (50-83.3\%) [1,3] due to an anatomic predisposition, having a lower coronary height and smaller aortic sinus of Valsalva. The estimated risk cut off for the former is $10-12 \mathrm{~mm}$, while for the latter is $30 \mathrm{~mm}[1,5,6]$. Moreover, a severe degree of calcification of the aortic valve apparatus is an important risk factor $[1,3,7]$. The valve-in-valve procedure is associa-

Address for correspondence: Marco Toselli, MD, Division of Cardiology, Azienda Ospedaliera Universitaria di Parma, Via Gramsci 14, 43216 Parma, Italy, tel: +39 340 7968618, fax +49 30450 565910, e-mail: marco.toselli2@gmail.com Received: 1.08.2018 Accepted: 20.11.2018 


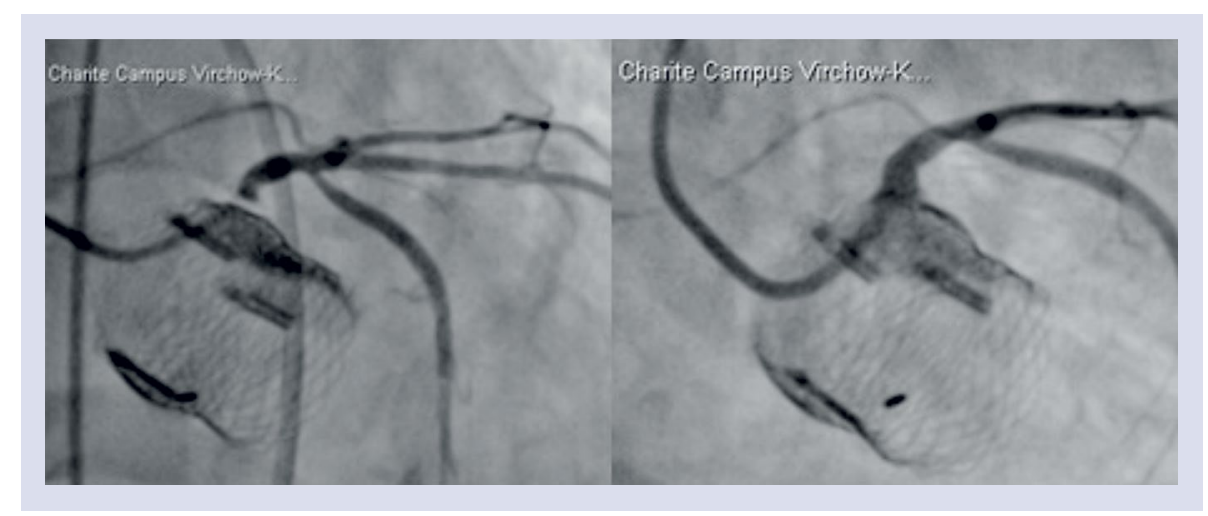

Figure 1. Coronary angiogramm showing a slit like narrowing of the ostial left main originating and final result after complex percutaneous coronary intervention with stent-in-stent implantation.

ted with a higher risk of CO [1]. Data regarding association between valve type and $\mathrm{CO}$ are contradictory: balloon-expandable devices were more often involved [3], but Jabbour et al. [1] in a recent large case-series reported a higher incidence with self-expandable devices possibly due to persistent expansion.

Clinical presentation of DCO is heterogeneous, ranging from stable angina and ventricular arrhythmias to acute myocardial infarction and cardiac arrest $[1,3]$. Severe and persistent hypotension after the TAVR procedure, not necessarily associated with electrocardiogram changes, may be a leading symptom [2]. Left main artery is most often involved (83.3-92.1\%) compared to the right coronary artery $(12.5-26.3 \%)[1,3]$. The underlying pathogenetic events triggering $\mathrm{CO}$ are multiple: thrombotic embolism, obstruction due to native or bioprosthesis valve leaflets, mechanical obstruction by the valve strut or calcium dislocation $[1,5,6,8]$. In many cases however, the etiology remains unclear.

The overall mortality rate in hospitalized patients is up to $50 \%$. Urgent percutaneous coronary intervention is recognized to be the favored treatment strategy with a successful outcome in $74.3-91.3 \%$ of cases $[1,3,4]$. In a systematic review by Ribeiro et al. [3] $13.4 \%$ of the patients required implantation of a second stent (stent-instent) because of persistent and elevated compression. In stable clinical settings, coronary computed tomography angiography maybe considered the first line option to rule out $\mathrm{CO}$ [1].

Clinical suspicion and history of TAVR should be taken into account for early recognition of DCO. Stent implantation through the meshes of the biological valve is a high risk and challenging procedure but can lead to a successful outcome.

\section{Conflict of interest: None declared}

\section{References}

1. Jabbour RJ, Tanaka A, Finkelstein A, et al. Delayed Coronary Obstruction After Transcatheter Aortic Valve Replacement. J Am Coll Cardiol. 2018; 71(14): 1513-1524, doi: 10.1016/j. jacc.2018.01.066, indexed in Pubmed: 29622157.

2. Krackhardt F, Kherad B, Krisper M, et al. Low permanent pacemaker rates following Lotus device implantation for transcatheter aortic valve replacement due to modified implantation protocol. Cardiol J. 2017; 24(3): 250-258, doi: 10.5603/CJ.a2017.0024, indexed in Pubmed: 28248410.

3. Ribeiro HB, Nombela-Franco L, Urena M, et al. Coronary obstruction following transcatheter aortic valve implantation: a systematic review. JACC Cardiovasc Interv. 2013; 6(5): 452-461, doi: 10.1016/j.jcin.2012.11.014, indexed in Pubmed: 23602458.

4. Durmaz T, Ayhan H, Keles T, et al. Left main coronary artery obstruction by dislodged native-valve calculus after transcatheter aortic valve replacement. Tex Heart Inst J. 2014; 41(4): 414-417, doi: 10.14503/THIJ-13-3410, indexed in Pubmed: 25120396.

5. Freixa X, Bonan R, Asgar AW. Unusual coronary occlusion post transcatheter aortic implantation: the importance of clinical assessment. Can J Cardiol. 2013; 29(8): 1014.e5-1014.e6, doi: 10.1016/j.cjca.2012.09.018, indexed in Pubmed: 23265096.

6. Webb JG. Coronary obstruction due to transcatheter valve implantation. Catheter Cardiovasc Interv. 2009; 73(7): 973, doi: 10.1002/ccd.22105, indexed in Pubmed: 19455693.

7. Kukucka M, Pasic M, Dreysse S, et al. Delayed subtotal coronary obstruction after transapical aortic valve implantation. Interact Cardiovasc Thorac Surg. 2011; 12(1): 57-60, doi: 10.1510/ /icvts.2010.252866, indexed in Pubmed: 21098421.

8. Neuss M, Kaneko H, Tambor G, et al. Fatal thrombotic occlusion of left main trunk due to huge thrombus on Prosthetic aortic valve after transcatheter aortic valve replacement. JACC Cardiovasc Interv. 2016; 9(21): 2257-2258, doi: 10.1016/j. jcin.2016.08.027, indexed in Pubmed: 27744041. 